providers in high-cost settings. There are no systematic studies of community pharmacy use by parents in the UK.

Aim To describe the use of community pharmacist minor ailment service (MAS) by parents in a single Borough

Methods A CCG service level agreement was in place for community pharmacists MAS. Mandatory completion of every consultation was carried out over a 12 month period.Age, presenting illness, time to consultation and feedback on the service were collected. Descriptive analysis of data.

Results 6974 consultations by 4174 patients over 12 month period (02/2013 to 02/2014) involving 47 Pharmacies with the provision of 70 over the counter (OTC) medications for 20 ailments.More than half $(57 \%, \mathrm{n}=2,379)$ of the patients below the age of $163593.52 \%$ of the consultations occurred in infants of which only $2 \%$ (143) were signposted to either the GP or accident and emergency.Fever, Hay fever and sore throat were the 3 most common ailments treated at the pharmacy, and these accounted for 58\% of total consultations. In addition, $85 \%(n=3535)$ of patients were seen not more than twice with only $19(0.5 \%)$ patients using the scheme more than 10 times within the 12 month period. Notably, 96\% of all patients were seen within $10 \mathrm{~min}$ (and $69 \%<5 \mathrm{~min}$ ) of arrival. Of the 4302 respondents, 99\% (4273) fed back they would reuse the service again.

Conclusions Community pharmacists contribute a valuable service for this group of children and may allow for more appropriate demand management in the community for less severe illness. There is potential for further involvement of paediatricians in supporting community pharmacist. This study needs to be repeated in other sites.

\section{G70 SUDDEN UNEXPECTED DEATH IN INFANCY/CHILDHOOD MULTIDISCIPLINARY SIMULATION STUDY DAY EXPERIENCE}

D Beasley, T Hildebrandt. Paediatrics, Abertawe Bro Morgannwg University Health Board, Swansea, UK

\subsection{6/archdischild-2018-rcpch.68}

Introduction Thankfully sudden unexpected death in infancy/ childhood (SUDI/C) is rare but a tragedy for parents. Clinicians have often very limited experience in dealing with unexpected death in childhood. We designed a multidisciplinary simulation study day to equip senior paediatric trainees with knowledge and skills required to lead child death review process and support parents.

Method The study day combined interactive lectures from multi-agency experts including police, social services, a community paediatrician and pathologist. This was followed by simulation of resuscitation, communication with parents and police as well as a simulation of a SUDI/C meeting. Actors were used to enhance fidelity. All participants (17) were required to fill in questionnaires pre- and post study day attendance.

Results Evaluation of pre-study day questionnaires confirmed lack of experience and knowledge about SUDI/C process. Post study day feedback was overwhelmingly positive in terms of credibility and usefulness.

Conclusion There is a lack of knowledge and experience regarding SUDI/C amongst senior paediatric trainees. Multidisciplinary simulation is a useful tool to prepare senior trainees and junior consultants to deal with SUDI/C process effectively and sensitively.

Recommendation We are currently in the process of organising a second study day open to a variety of professionals.

\section{G71 WITH A LITTLE HELP FROM YOUR FRIENDS - A COLLABORATIVE SIMULATION PROGRAMME IMPROVES PAEDIATRIC TRAINEES' CONFIDENCE IN MANAGING COMPLEX MENTAL HEALTH PRESENTATIONS}

${ }^{1} \mathrm{M}$ Shiri-Feshki, ${ }^{2} \mathrm{~N}$ Mullen, ${ }^{1} \mathrm{~B}$ Owen. ${ }^{1}$ Medical Education Department, St Nicholas Hospital, Northumberland, Tyne and Wear NHS Foundation Trust, Newcastle upon Tyne, UK; ${ }^{2}$ Simulation in the North-East, Sunderland Royal Hospital, City Hospitals Sunderland Foundation NHS Trust, Sunderland, UK

\subsection{6/archdischild-2018-rcpch.69}

Objectives Paediatric trainees encounter complex acute mental health presentations infrequently. We developed and assessed the impact of a collaborative Paediatric Mental Health simulation training programme for senior paediatric trainees (ST68).

Methodology The one day course was designed by a multiprofessional and multidisciplinary team comprising:

Children and Adolescent Mental Health Consultants and senior trainees

Senior Acute psychiatry liaison nurses

Paediatric Emergency Medicine Consultant

Paediatric Simulation Lead

Educationalists from Northumbria University

Based on curriculum needs and the experience of the faculty we designed three challenging scenarios:

A young person with anorexia nervosa and diabetes refusing treatment

An agitated young person with challenging behaviour

A young person refusing investigation and treatment following a substantial paracetamol overdose

We used medical and nursing students as simulated patients following careful pre-brief.

Using anonymous self-rating questionnaires we assessed the participants' confidence levels before, immediately after and 4 month after the course. We linked the pre and post-course questionnaires using the last three digits of the participants' post-codes.

Results We received 18 pre and immediate post course questionnaires. Of 18 questionnaires 15 were able to be matched immediately after the course. We also received 5 matched questionnaires after 4 months. The differences were calculated and the percentage of the means plotted.

Immediate effects The results showed an improvement in confidence in almost all domains. There was particular improvement in the domains labelled 'clinical patient care' and 'collaborative practice'.

Medium effects (after 4 months):

The results after 4 months showed sustained improvement in learners' confidence in the majority of domains.

Conclusion Our programme has resulted in an immediate and sustained improvement in paediatric trainees' confidence in managing complex acute mental health presentations.

We attribute our success in meeting this crucial yet previously unmet need to collaboration between senior colleagues from paediatrics, psychiatry and education. 